\title{
Extraction and Determination of Three Chlorophenols by Hollow Fiber Liquid Phase Microextraction - Spectrophotometric Analysis, and Evaluation Procedures Using Mean Centering of Ratio Spectra Method
}

\author{
Zarrin Es'haghi \\ Department of Chemistry, Faculty of Sciences, Payame Noor University, Mashhad, Iran \\ E-mail: z_eshaghi@pnu.ac.ir \\ Received October 16, 2010; revised November 25, 2010; accepted November 27, 2010
}

\begin{abstract}
A method termed hollow fiber liquid phase microextraction (HF-LPME) was utilized to extract three chlorophenols, 2-chlorophenol (2-CP), 2,4-dichlorophenol (2,4-DCP) and 2,4,6-trichlorophenol (2,4,6-TCP), separately from water. The extracted chlorophenols were then separated, identified, and quantified by UV-Vis spectrophotometry with photodiode array detection (UV-Vis/DAD). In the study, experimental conditions such as organic phase identity, acceptor phase volume, sample agitation, extraction time, acceptor phase $\mathrm{NaOH}$ concentration, donor phase $\mathrm{HCl}$ concentration, salt addition, and UV absorption wavelength were optimized. The statistical parameters of the proposed method were investigated under the selected conditions. The analytical characteristics of the method such as detection limit, accuracy, precision, relative standard deviation (R.S.D.) and relative standard error (R.S.E.) were calculated. The results showed that the proposed method is simple, rapid, accurate and precise for the analysis of ternary mixtures.
\end{abstract}

Keywords: Chlorophenol, Hollow Fiber Liquid Phase Microextraction (HF-LPME), UV-Vis Spectrophotometry, Photodiode Array Detector

\section{Introduction}

Cholorophenols (CPs) are chemical species known to be highly toxic and a potential threat to public health. CPs are used extensively as preservatives, fungicides, pesticides, disinfectants, and intermediates in many industries [1]. CPs are generated from phenols during the treatment of tap water with chlorine and are considered to be one of the most obnoxious contaminants [2,3] because they deteriorate taste and produce an unfavorable smell. Moreover, they are thought to be serious health hazards because they accumulate in moderate amounts and show high toxicity $[4,5]$. These hazardous materials are usually detected in human urine because of the intake of food and water containing CPs and other chlorinated substances as metabolites present in the environment $[6,7]$. In order to assess human exposure to CPs, a reliable and sensitive analytical method is required.
Many analytical methods, including capillary electrophoresis [7], high-performance liquid chromatography (HPLC) $[8,9]$ and gas chromatography (GC) $[10,11]$, are available for the determination of CPs in human urine samples. Methods such as ultraviolet-visible spectrophotometry may be profitable for some applications instead of expensive methods such as HPLC and CEC.

Despite highly selective separation and sensitive instrumentation for quantification, the direct introduction of analytes to the instruments is not usually compatible with environmental determinations.

The sample preparation step in an analytical process typically consists of an extraction procedure that results in the isolation and enrichment of components of interest from a sample matrix.

Classical extraction procedures consume large amounts of solvents, thus creating environmental and occupational hazards, and often provide very little selectivity. 
These results emphasize the need for reduction of solvent use, automation, and miniaturization.

Microextraction techniques are the result of looking for the miniaturization of classical extraction techniques in order to expend minimum analysis time and chemicals [12]. In recent years, efforts have been made towards the miniaturization of the traditional liquid-liquid extraction. These techniques, based on the contact between two immiscible liquids, fall into two main categories, namely single-drop microextraction (SDME) $[13,14]$ and hollow fiber liquid phase microextraction (HF-LPME) $[15,16]$.

Hollow fiber liquid-phase microextraction (HF-LPME) was introduced by Pedersen-Bjergaard and Rasmussen to improve the stability and reliability of single drop liquid-phase microextraction (LPME). In this microextraction technique, a water-immiscible organic solvent was immobilized in the pores of a porous hollow fiber, and formed a supported liquid membrane.

The lumen of the hollow fiber was filled with micro-liter amounts of an acceptor phase. The analytes were extracted from the aqueous donor phase into the organic solvent, and then captured into the acceptor phase. After extraction, the extracted phase was directly introduced to the analytical instruments [15]. Three phase HF has been applied to the determination of CPs in this research.

\section{Experimental Section}

\subsection{Reagents and Samples}

2-Chlorophenol (2-CP), 2,4-dichlorophenol (2,4-DCP) and 2,4,6-trichlorophenol (2,4,6-TCP) were purchased from Sigma-Aldrich GmbH (Steinheim, Germany). Methanol and acetone and the other solvents used for this study were of HPLC grade. 1-Octanol was purchased from Merck, Darmestadth, Germany.

Stock solutions of the chlorophenols were prepared by dissolving each chlorophenol in methanol to obtain 100 $\mathrm{mg} \cdot \mathrm{mL}^{-1}$ solutions. Aliquots of these stock solutions were diluted with water to prepare standard working solutions at a concentration of $100 \mathrm{ng} / \mathrm{mL}$. All the other reagents and solvents used were of analytical reagent grade.

A Q3/2 Accurel polypropylene hollow fiber membrane (600 $\mu \mathrm{m}$ i.d., $200 \mu \mathrm{m}$ wall thickness, $0.2 \mu \mathrm{m}$ pore size) was purchased from Membrana GmbH (Wuppertal, Germany). The hollow fiber was cut into $4.0 \mathrm{~cm}$ segments, cleaned with acetone, and dried before use.

De-ionized water was purified in a Milli-Q water purification system (Millipore, Bedford, MA, USA). Field samples, a reservoir and a tap water sample, were collected from Mashhad, Iran.

\subsection{Extraction Process and Initial Conditions}

A $14-\mathrm{mL}$ aqueous solution $(0.1 \mathrm{M} \mathrm{HCl})$ containing all analytes at concentrations of $100 \mathrm{ng} / \mathrm{mL}$ each and a $1 \mathrm{~cm}$ stir bar were placed in a 16-mL sample vial previously loaded with $1 \mathrm{~g}$ of $\mathrm{NaCl}$. The solution was stirred at 1000 rpm for 2 min to dissolve the $\mathrm{NaCl}$. Acceptor solution $(12.5 \mu \mathrm{L}, 0.1 \mathrm{M} \mathrm{NaOH})$ was withdrawn into the microsyringe. The needle tip of the microsyringe was then inserted into the $5.0 \mathrm{~cm}$ hollow fiber, and the fiber was filled with the acceptor phase. Before that, the fiber pores were impregnated with organic solvent. After impregnation, excess organic solvent was carefully removed from the lumen of the fiber. Then, the fiber was moved to the aqueous sample solution. The magnetic stirrer was set at a speed of $500 \mathrm{rpm}$. The setup arrangement is illustrated in Figure 1. After extracting for $40 \mathrm{~min}$, the stirrer was stopped. All acceptor phase (about $12 \mu \mathrm{L}$ ) was withdrawn back into the microsyringe. Finally, the acceptor phase was injected into a UV-Vis cell and then was diluted to $2.0 \mathrm{~mL}$ with acceptor phase solution and introduced into the spectrophotometer for further analysis.

\section{Results and Discussion}

In this experiment, the chlorophenols were extracted from an acidified donor phase (sample solution) into an organic phase, which impregnated the pores of a hollow fiber. The chlorophenols were then converted into chlorophenolate anion by extracting the chlorophenols from the organic phase into an alkaline acceptor phase (enriched extract). The extracted analytes were then quantified by UV-Vis spectrophotometer/DAD.

\subsection{Optimization of Variables}

The microextraction conditions for the three chlorophe-

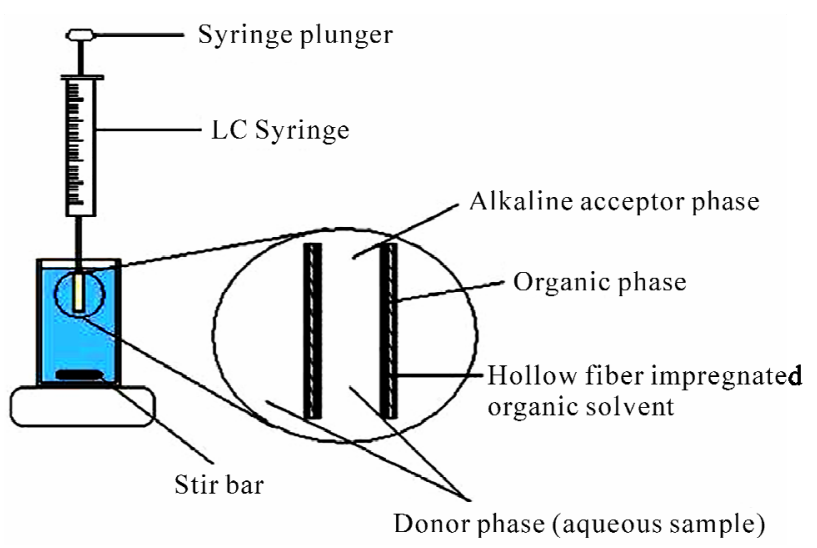

Figure 1. Hollow fiber liquid phase microextraction apparatus. 
nols must be optimized. In this purpose the univariant method was conducted to pursue the optimal experimental conditions for the microextraction procedure. Analytical parameters, including organic phase identity, acceptor phase volume, stirring rate, extraction time, acceptor phase $\mathrm{NaOH}$ concentration, donor phase $\mathrm{HCl}$ concentration, and salt addition were investigated and identified to improve efficiency. The conditions for LLLME are as follows: $14 \mathrm{~mL}$ of $6.0 \mathrm{M} \mathrm{HCl}, 1.5 \mathrm{~g}$ of $\mathrm{NaCl}(\mathrm{s})$ as the donor phase, $15 \mu \mathrm{L}$ of $0.1 \mathrm{M} \mathrm{NaOH}$ as the acceptor phase, 1,2,4-trichlorobenzene/1-octanol (70/30) as the organic phase, $900 \mathrm{rpm}$ stirring speed, and $45 \mathrm{~min}$ extraction time.

\subsection{Selection of Organic Solvent}

The selection principles for a suitable organic solvent are as follows: First, the solvent should be easily immobilized in the pores of the polypropylene hollow fiber. Even more important is that the solvent must be of low volatility and immiscible with water. Most important of all, the solubility's of the analytes in the organic solvent must be higher than in the donor phase. Some proper organic solvents were examined for extracting chlorophenols. Accordingly, o-xylene (OX), p-xylene (PX), octanol (OC), and 1,2,4-trichlorobenzene (TCB) were tested. The results shown in Figure 2 indicate how extraction efficiencies were achieved with these aromatic solvents.

Two xylenes with non-polar functional groups showed acceptable extraction ability, presumably by forming induced dipoles by ring-electrons. Trichlorobenzene also has high extraction efficiency because the molecular structure of tri-chlorobenzene is very similar to those of the chlorophenols, except that the low polarity of trichloro benzene is such as to cause low extraction efficiencies for monochlorophenols. To improve extraction for all the chlorophenols, extractions utilizing various solvent mixtures $(\mathrm{v} / \mathrm{v}=1 / 1)$ were tested. As trial results indicated, the extractions of toxic polychlorophenols were more effective with trichlorobenzene, but 1-octanol was completely compatible with the polypropylene hollow fiber and thus use of solvent mixtures achieves better reproducibility and precision. Therefore 1,2,4-trichlorobenzene/octanol (70/30) was selected as the optimal organic phase.

\subsection{Effect of Extraction Time}

LLLME is dependent on equilibrium rather than exhaustive extraction [17]. The results obviously indicate that adequate time must be allowed for the system to reach equilibrium in the partitioning of analytes between the donor and acceptor phases (see Figure 3). However, when considering matching the extraction time with the duration of spectroscopic analysis, an extraction period of 45 min was chosen for subsequent extractions.

\subsection{Effect of Acceptor Phase Volume}

The acceptor phase was a basic solution $(0.1 \mathrm{M} \mathrm{NaOH})$. Generally, in the three-phase LLLME systems, a smaller volume of acceptor phase involves a higher analyte concentration (or enrichment) in the acceptor phase [18]. However, the important factor for LLLME is not concentration, but the total mass of the analytes in the acceptor phase. Accordingly, the acceptor phase should be of large volume to promote analyte transport to the acceptor phase. The acceptor phase volume was examined over the range of $7-17 \mu \mathrm{L}$. As trial results indicated, 15.0 $\mu \mathrm{L}$ of acceptor phase provided superior operation and this volume was used for subsequent extractions.

\subsection{Effect of the Donor Phase PH on the Extraction}

To extract the chlorophenol, a weak acid, into the organic

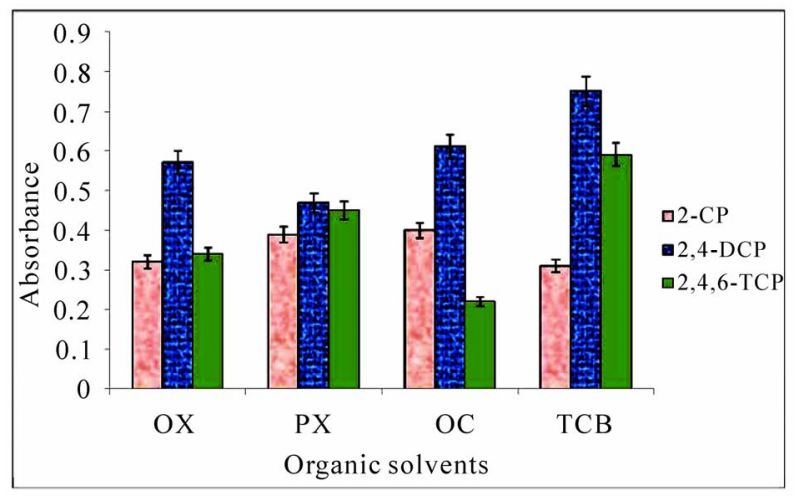

Figure 2. Effect of organic solvent on the extraction procedure.

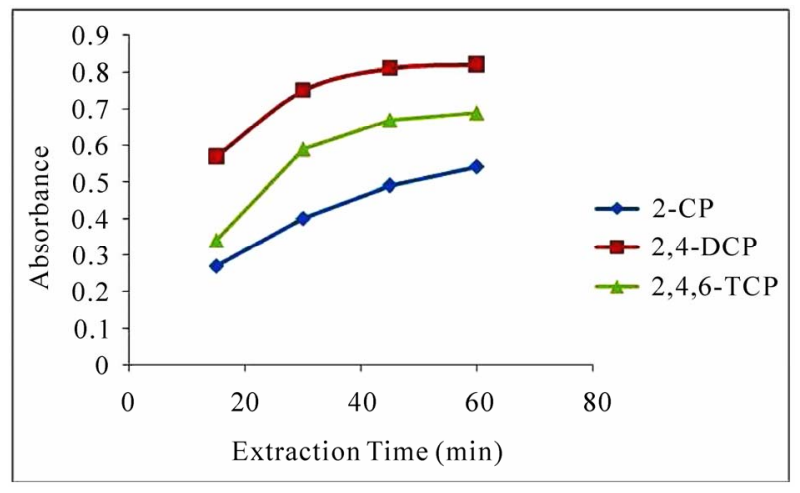

Figure 3. Effect of extraction time on the method efficiency. 
phase from the donor phase, the $\mathrm{pH}$ of the donor phase was acidified to convert the analytes into their molecular (i.e., uncharged) form [19].

As trial results indicated, the extraction efficiencies of the chlorophenols varied slightly with $\mathrm{HCl}(\mathrm{aq})$ concentration of the donor phase from $0.01 \mathrm{M}$ to $0.5 \mathrm{M}$. The pKa values of the chlorophenols studied range from 6.23 to 8.49. Theoretically, a $\mathrm{pH}$ value of the donor phase of 4.0 would be sufficiently acidic. In order to deal with potential matrix interference in real field samples, $\mathrm{HCl}$ solution with $\mathrm{pH}=3$ was used.

\subsection{Effect of Acceptor Phase PH on the Extraction}

Chlorophenols are weakly acidic in character, so the acceptor solution must be sufficiently alkaline to convert them to the ionic form in order to extract them from the organic phase. On examining $\mathrm{NaOH}(\mathrm{aq})$ concentrations from $0.01 \mathrm{M}$ to $1 \mathrm{M}$, the results indicate that $0.05 \mathrm{M}$ $\mathrm{NaOH}(\mathrm{aq})$ is satisfactory. Nevertheless, it is not of sufficiently high alkalinity for the extraction of real field samples. Many contaminants could be extracted into the acceptor phase from a real field sample matrix; these contaminants could neutralize the basicity of the acceptor phase. Because of this, 0.1 M NaOH(aq) was selected as the acceptor phase.

\subsection{Effect of Agitation Speed}

Agitation of the donor solution reduces the required extraction time and increases extraction efficiency. Stirring provides fresh donor solution for the organic phase to extract and reduces the effect of the stationary boundary layer zone (Nernestian layer) produced close to the organic phase; these factors promote analyte transport from the donor phase to the organic phase [20].

However, agitation in excess of the optimal stirring rate may also cause lower extraction efficiency because the hollow fiber is vibrated by the surrounding turbulent flow and air bubbles reduced absorption for each analyte and a decrease in the precision of the method [21].

To evaluate the effect of stirring, donor solution was extracted at varying stirring rates (300 - $1000 \mathrm{rpm}$ ). It was found that the extraction efficiencies of these chlorophenols increased at higher stirring rates and reached a maximum at $900 \mathrm{rpm}$. Consequently, $900 \mathrm{rpm}$ was chosen for subsequent extractions.

\subsection{Effect of Salt Addition}

Adding salt to the analytes may have contradictory effects. First, one expects a positive effect from salting out when the ionic strength of the donor phase is increased. This is due to the decrease in the solubility of the analytes in the aqueous phase and enhances their partitioning into the organic phase. Secondly, a negative effect is attributed to changes in the physical properties of the Nernst diffusion film, which results in reducing the rate of diffusion of the analytes from the donor phase into the organic phase. In addition, the salting out phenomenon would also reduce the solubility of organic solvent in water, causing the organic phase to exhaustively wall off the acceptor phase from the donor phase, a quite desirable result.

Therefore, 0.0 - $2.0 \mathrm{~g}$ of $\mathrm{NaCl}(\mathrm{s})$ was added to $14 \mathrm{~mL}$ of the donor phase to determine the effect of salt addition. As the experimental results indicated, the addition of 1.5 g $\mathrm{NaCl}(\mathrm{s})(9.7 \%, \mathrm{w} / \mathrm{w})$ of sodium chloride optimally enhanced the extraction of the chlorophenols in water. This quantity of $\mathrm{NaCl}$ was therefore used in subsequent experiments.

\subsection{Quantitative Aspects}

Absorbance measurements at the band maxima of the UV spectra obey the linear Beer's law more accurately than measurements off the band maxima. The UV spectra of the analytes can be utilized to identify target analytes in the UV-Vis spectrophotometer. Accordingly, each extracted chlorophenol was quantified at its own maximum adsorption wavelength (as shown in Figure 4).

The chlorophenols were determined under selected experimental conditions to assess repeatability, linearity, coefficient of determination, and detection limit. The results are shown in Table 1.

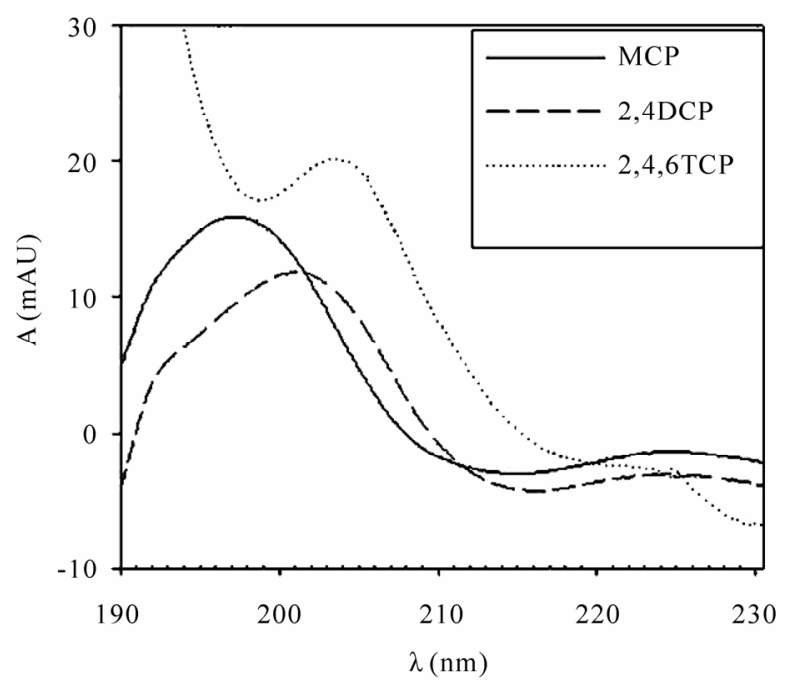

Figure 4. UV spectra of the various analytes, obtained with Diod Array Detector (DAD). 
Table 1. Method performance.

\begin{tabular}{ccccccc}
\hline Compound & $\begin{array}{c}\text { UV Wavelengh } \\
(\mathrm{nm})\end{array}$ & $\begin{array}{c}\text { Linear range } \\
(\mathrm{ng} / \mathrm{mL})\end{array}$ & $\mathbf{R}^{2}$ & $\begin{array}{c}\mathbf{L O D}^{\mathbf{a}} \\
(\mathrm{ng} / \mathrm{mL})\end{array}$ & $\left.\mathbf{R S D}^{\mathbf{2}}\right)^{\mathbf{b}}$ & $\left.\mathbf{R S D}^{\mathbf{2}}\right)^{\mathbf{c}}$ \\
\hline 2-CP & 197 & $\mathbf{0 . 5 - 1 0 0}$ & $\mathbf{0 . 9 9 7 8}$ & 01 & 7.3 & 3.8 \\
2,4-DCP & $201 \mathrm{~nm}$ & $\mathbf{0 . 5 - 1 0 0}$ & $\mathbf{0 . 9 9 9 1}$ & 0.08 & 3.5 & 2.7 \\
2,4,6-TCP & $204 \mathrm{~nm}$ & $\mathbf{0 . 5 - 1 0 0}$ & $\mathbf{0 . 9 9 8 6}$ & 0.09 & 3.9 & 2.7 \\
\hline
\end{tabular}

a) LODs are calculated as three times the standard deviation of seven replicated runs of a mixture containing $0.5 \mathrm{ng} \cdot \mathrm{mL}^{-1}$ each of the compound; b) Data are based on the absorbance of seven replicated runs of a mixture containing $0.5 \mathrm{ng} \cdot \mathrm{mL}^{-1}$ each of the compounds; c) Data is based on the absorbance of seven replicated runs of a mixture containing $5 \mathrm{ng} \cdot \mathrm{mL}^{-1}$ each of the compound.

Linearity was established over the concentration range of $0.5-150 \mathrm{ng} / \mathrm{mL}$. The coefficient of determination $\mathrm{r}^{2}$ varied from 0.9978 to 0.9991 . The limit of detection (LOD) values were calculated as three times the standard deviation of seven replicate runs of water spiked with analytes at $1.0 \mathrm{ng} / \mathrm{mL}$ each. The relative standard deviation (RSD) values were calculated for seven replicate runs, and these values were less than $7.4 \%$.

\subsection{Real Sample Analysis}

Two samples, waste-water (collected from an industrial center near Mashhad, Iran) and tap water (from Mashhad, Iran), were analyzed to demonstrate the practical applicability of this technique.

These two samples were filtered with a Milli-Q filtering system (Millipore), and the tap water sample was overdosed with sodium thiosulfate $(80 \mu \mathrm{g} / \mathrm{mL})$ to neutralize residual chlorine or hypochlorite. The analytical results of these samples indicated that the tap water sample is free from chlorophenols, but a target analyte is present in the waste-water sample. The detected analyte, whose concentration is $0.8 \mathrm{ng} / \mathrm{mL}$, coincides with $2,4,5-$ trichlorophenol.

For recovery, this LLLME method depends on equilibrium rather than exhaustive extraction.

Instead of absolute recoveries, relative recoveries determined as the ratio of the real field sample outcome to that of the deionized water sample are summarized in Table 2. These results indicate that the relative recoveries of chlorophenols from the spiked waste-water and tap water samples (performed in three replicates on 0.5 $\mathrm{ng} / \mathrm{mL}$ of each compound spiked) were $94.1 \%-101.4 \%$ and $87.8 \%-99.2 \%$, respectively.

\subsection{Chemometrics Evaluation of the Method}

The main problem of spectrophotometric multicomponent analysis is the simultaneous determination of two or more compounds in the same mixtures without preliminary separation.
Table 2. Relative recovery (\%) of practical water samples $(\mathbf{N}=3)$.

\begin{tabular}{ccc}
\hline Compound & Waste $^{\mathrm{A}}$ water & Tap $^{\mathrm{B}}$ water \\
\hline 2-CP & 94.1 & 99.2 \\
2,4-DCP & 99.9 & 87.8 \\
2,4,6-TCP & 101.4 & 99.0 \\
\hline
\end{tabular}

A) The sample was obtained from an industrial center, Mashhad, Iran; B) The tap water was from Mashhad, Iran.

Several spectrophotometric determination methods have been used for resolving mixtures of compounds with overlapping spectra. When these methods are compared with each other, the range of application of derivative spectrophotometry is more reliable with respect to utility and sensitivity than normal spectrophotometry. Other spectrophotometric methods, such as partial least squares regression (PLSR), principal component regression (PCR), multi-wavelength linear regression analysis (MLRA), H-point standard addition method (HPSAM) for binary and ternary mixtures have been utilized [2225].

Recently, Bahram et al. proposed a new spectrophotometric method for the simultaneous determination of ternary mixtures, without prior separation steps. This method is called the successive derivative ratio spectra $[26,27]$. The method is based on the successive derivatives of ratio spectra in two steps. The method was evaluated by model data and also by application to the simultaneous spectrophotometric determination of cobalt, nicked and zinc based on their complexes with 1-(2pyridylazo)2-naphthol in micellar media as experimental data .

In our research this new and very simple method was developed for the simultaneous determination of ternary chlorophenols mixtures, with prior separation steps. This method is based on the mean centering of ratio spectra. This method eliminates derivative steps and therefore signal-to-noise ratio is enhanced. 


\section{Modeling}

\subsection{Validation of the Method}

Validation of the method with model data to demonstrate the analytical applicability of the proposed method for the analysis of ternary mixtures, three spectra were created. The three curves form model of the overlapping spectra of three compounds $X, Y$ and $Z$ in the range 190 230 nm (Figure 4).

The mathematical explanation of the procedure was illustrated by the authors. After modeling procedure, the method has been applied to the simultaneous analysis of ternary mixtures of chloprophenols. The analytical characteristics of the method such as detection limit, accuracy, precision, relative standard deviation (R.S.D.) and relative standard error (R.S.E.) was calculated. The results showed that the proposed method is accurate and precise method for analysis of ternary mixtures (see Tables 3 and 4).
Limit of detection of the method for determination of chloprophenols in their ternary mixtures (defined as the concentration equivalent to three times the standard deviation of five replicate measurements of the blank) are also shown in Table 4.

The effect of divisor concentration on the analytical parameters such as detection limit, slope, intercept and correlation coefficient of the calibration equations was tested. It was observed that changing the concentration of divisors in their linear calibration range had no significant effect on the analytical parameters.

Therefore, a normalized spectrum of each of the analytes was used as divisor spectrum in the proposed method.

In order to obtain the accuracy and precision of the method, several synthetic mixtures with different concentration ratios of chlorophenols were analyzed using the proposed method.

The prediction error of a single component in the mixtures was calculated as the relative standard error (R.S.E.)

Table 3. Results for several experiments for analysis of chlorophenols in ternary mixtures in different concentration ratios by proposed method, HF-LPME-MCRS.

\begin{tabular}{|c|c|c|c|c|c|c|c|c|}
\hline \multicolumn{3}{|c|}{ Conc. (ng/mL) (in mixture) } & \multicolumn{3}{|c|}{ Estimated Conc. (ng/mL) } & \multicolumn{3}{|c|}{ Recovery (\%) } \\
\hline 2-CP & 2,4-DCP & 2,4,6-ТCP & 2-CP & 2,4-DCP & 2,4,6-ТСР & $2-\mathrm{CP}$ & 2,4-DCP & 2,4,6-ТCP \\
\hline 0.2 & 0.9 & 0.1 & 0.2 & 0.81 & 0.15 & 100 & 90 & 100 \\
\hline 0.4 & 0.8 & 0.2 & 0.41 & 0.93 & 0.28 & 102.5 & 98.75 & 90 \\
\hline 0.4 & 0.8 & 1 & 0.33 & 0.73 & 1 & 82.5 & 98.75 & 100 \\
\hline 0.8 & 0.2 & 0.3 & 1 & 0.24 & 0.29 & 125 & 110 & 95 \\
\hline 1 & 0.1 & 0.6 & 0.96 & 0.1 & 0.63 & 96 & 100 & 95 \\
\hline 0.9 & 0.4 & 0.2 & 0.9 & 0.45 & 0.2 & 100 & 95 & 100 \\
\hline 0.6 & 0.6 & 0.1 & 0.57 & 0.61 & 0.1 & 95 & 101.66 & 100 \\
\hline 0.9 & 0.5 & 0.4 & 0.91 & 0.59 & 0.33 & 101.11 & 98 & 82.5 \\
\hline Mean recovery \% & & & & & & 100.51 & 100.37 & 97.92 \\
\hline R.S.E. single \% & & & & & & 9.879 & 6.751 & 5.862 \\
\hline R.S.E. total \% & & & & & & & & 7.5 \\
\hline
\end{tabular}

Table 4. Analytical aspects of results in ternary mixture by HF-LPME-MCRS methods.

\begin{tabular}{|c|c|c|c|c|c|c|}
\hline Analyte & Wavelength max & Calibration slope & $\begin{array}{c}\text { Calibration } \\
\text { intercept }\end{array}$ & $\mathbf{R}^{2}$ & $\begin{array}{c}\text { Linear range } \\
\left(\mathrm{ng} \cdot \mathrm{mL}^{-1}\right)\end{array}$ & $\begin{array}{c}\text { LOD } \\
\left(\mathrm{ng} \cdot \mathrm{mL}^{-1}\right)\end{array}$ \\
\hline 2-CP & 197 & 9.99 & -0.0066 & 0.9999 & $0.01-100$ & 0.007 \\
\hline 2,4-DCP & 201 & 86.26 & -14.446 & 0.9960 & $0.01-100$ & 0.008 \\
\hline s2,4,6-TCP & 204 & 8.27 & +0.0139 & 0.9998 & $0.01-100$ & 0.008 \\
\hline
\end{tabular}


of the prediction concentration. Table 4 also shows the reasonable single and total relative errors for such system.

\section{Conclusions}

The results obtained in this work indicate the application of hollow fiber three liquid phase microextraction of chlorophenols from water samples, with the obvious advantages of higher enrichment factors and lower detection limits. This technique is simple, economical, safe for the examiner, rapid, sensitive and easy to understand and apply.

The application of UV-Vis spectrophotometry for the simultaneous determination of analytes in water samples is feasible by using homemade software. This technique consists of a fast, inexpensive procedure and provides an easy method with which to assay the target analytes in environmental samples.

\section{References}

[1] R. C. C. Wegman and A. W. M. Hofster, "Chlorophenols in Surface Waters of the Netherlands (1976-1977),” Water Research, Vol. 13, 1979, pp. 651-657. doi:10.1016/0043-1354(79)90015-0

[2] J. Lezamiz and J. A. Jönsson, "Development of a Simple Hollow Fibre Supported Liquid Membrane Extraction Method to Extract and Preconcentrate Dinitrophenols in Environmental Samples at ng $\cdot \mathrm{L}(-1)$ Level by Liquid Chromatography," Journal of Chromatography A, Vol. 1152, No. 1-2, 2007, pp. 226-233. doi:10.1016/j.chroma.2006.11.104

[3] T. Mathialagan and T. Viraraghavan, "Biosorption of Chlorophenols: A Review,” International Journal of Environment and Pollution, Vol. 34, No. 1-4, 2008, pp. 164-194. doi:10.1504/IJEP.2008.020790

[4] A. Ribeiro, M. H. Neves, M. F. Almeida, A. Alves and L. Santos, "Direct Determination of Chlorophenols in Landfill Leachates by Solid-Phase Micro-Extraction-Gas Chromatography-Mass Spectrometry," Journal of Chromatography A, Vol. 975, No. 2, 2002, pp. 267-274. doi:10.1016/S0021-9673(02)01280-3

[5] F. Bianchi, M. Careri, C. Mucchino and M. Musci, "Improved Determination of Chlorophenols in Water by Solid-Phase Microextraction Followed by Benzoylation and Gas Chromatography with Electron Capture Detection,” Chromatographia, Vol. 55, 2002, pp. 595-600. doi:10.1007/BF02492907

[6] M. C. Quintana and L. Ramos, "Sample Preparation for the Determination of Chlorophenols," Trends in Analytical Chemistry, Vol. 27, No. 5, 2008, pp. 418-436. doi:10.1016/j.trac.2008.03.009

[7] P. Verónica, A. Juan, G. Venerando and A. Ana, "Monitoring Chlorophenols in Industrial Effluents by Solid-
Phase Microextraction-Gas Chromatography-Mass Spectrometry," International Journal of Environmental Analytical Chemistry, Vol. 87, No. 3, 2007, pp. 159-175. doi:10.1080/03067310600847252

[8] Q. B. Cass, L. G. Freitas, E. Foresti, M. Zamariolli and M. H. R. Aminaovic, "Developmet of HPLC Method for the Analysis of Chlorophenosl in Samples from Anaerobic Reactors for Waste Water Treatment,” Journal of Liquid Chromatography \& Related Technologies, Vol. 23, No. 7, 2000, pp. 1089-1097. doi:10.1081/JLC-100101510

[9] T. Araki, M. Chiba, S. Tsunol and M. Tanaka, “Separation of Chlorophenols by HPLC and Capillary Electrochromatography Using beta-Cyclodextrin-bonded Stationary Phases,” Analytical Sciences, Vol. 16, No. 4, 2000, pp. 421-424. doi:10.2116/analsci.16.421

[10] L. W. Chung and M. R. Lee, "Evaluation of Liquid-Phase Microextraction Conditions for Determination of Chlorophenols in Environmental Samples Using Gas Chromatography-Mass Spectrometry without Derivatization," Talanta, Vol. 76, 2008, pp. 154-160. doi:10.1016/j.talanta.2008.02.018

[11] P. Tölgyessy, B. Vrana, M. Bartal, Z. Krascsenits and K. Šilhárova, "Determination of Chlorophenols in Sediments Using Ultrasonic Solvent Extraction Followed by Stir Bar Sorptive Extraction Coupled to TD-GC-MS," Chromatographia, Vol. 69, 2009, pp. 389-392. doi:10.1365/s10337-008-0877-y

[12] J. A. Jönsson and L. Mathiasson, "Liquid Membrane Extraction in Analytical Sample Preparation: I. Principles," Trends in Analytical Chemistry, Vol. 18, No. 5, 1999, pp. 318-325. doi:10.1016/S0165-9936(99)00102-8

[13] E. Psillakis and N. Kalogerakis, "Developments in Single-Drop Microextraction,” Trends in Analytical Chemistry, Vol. 21, No. 1, 2002, pp. 53-63. doi:10.1016/S0165-9936(01)00126-1

[14] M. A. Jeannot and F. F. Cantwell, "Solvent Microextraction into a Single Drop,” Analytical Chemistry, Vol. 68, 1996, pp. 2236-2240. doi:10.1021/ac960042z

[15] J. Lee, H. K. Lee, K. E. Rasmussen and S. Pedersen-Bjergaard, "Environmental and Bioanalytical Applications of Hollow Fiber Membrane Liquid-Phase Microextraction: A Review,” Analytica Chimica Acta, Vol. 624, No. 2, 2008, pp. 253-268. doi:10.1016/j.aca.2008.06.050

[16] K. E. Rasmussen and S. Pedersen-Bjergaard, "Developments in Hollow Fibre-Based, Liquid-Phase Microextraction," Trends in Analytical Chemistry, Vol. 23, No. 1, 2004, pp. 1-10. doi:10.1016/S0165-9936(04)00105-0

[17] C. C. Chen, M. B. Melwanki and S. D. Huang, "Liquid-Liquid-Liquid Microextraction with Automated Movement of the Acceptor and the Donor Phase for the Extraction of Phenoxyacetic Acids Prior to Liquid Chromatography Detection,” Journal of Chromatography A, Vol. 1104, No. 1-2, 2006, pp. 33-39. doi:10.1016/j.chroma.2005.11.122

[18] A. Sarafraz-Yazdi and Z. Es'haghi, "Liquid-Liquid-Liquid Phase Microextraction of Aromatic Amines in Water Using Crown Ethers by High-Performance Liquid Chroma- 
tography with Monolithic Column,” Talanta, Vol. 66, No. 3, 2005, pp. 664-669. doi:10.1016/j.talanta.2004.12.026

[19] A. Gjelstad, T. M. Andersen, K. E. Rasmussen and S. Pedersen-Bjergaard, "Microextraction across Supported Liquid Membranes Forced by $\mathrm{pH}$ Gradients and Electrical Fields,” Journal of Chromatography A, Vol. 1157, No. 1-2, 2007, pp. 38-45. doi:10.1016/j.chroma.2007.05.007

[20] W. Xie, J. Pawliszyn, W. M. Mullett and B. K. Matuszewski, "Comparison of Solid-Phase Microextraction and Liquid-Liquid Extraction in 96-Well Format for the Determination of a Drug Compound in Human Plasma by Liquid Chromatography with Tandem Mass Spectrometric Detection,” Journal of Pharmaceutical and Biomedical Analysis, Vol. 45, No. 4, 2007, pp. 599-608. doi:10.1016/j.jpba.2007.08.029

[21] A. S. Yazdi and Z. Es'haghi, “Two-step Hollow FiberBased, Liquid-Phase Microextraction Combined with High-Performance Liquid Chromatography: A New Approach to Determination of Aromatic Amines in Water," Journal of Chromatography A, Vol. 1082, No. 2, 2005, pp. 136-142. doi:10.1016/j.chroma.2005.05.102

[22] M. Palit, D. Pardasani, A. K. Gupta and D. K. Dubey, "Application of Single Drop Microextraction for Analysis of Chemical Warfare Agents and Related Compounds in Water by Gas Chromatography/Mass Spectrometry," Analytical Chemistry, Vol. 77, 2005, pp. 711-717. doi:10.1021/ac0486948
[23] E. Dinç and F. Onur, “Application of a New Spectrophotometric Method for the Analysis of a Ternary Mixture Containing Metamizol, Paracetamol and Caffeine in Tablets,” Analytica Chimica Acta, Vol. 359, No. 1-2, 1998, pp. 93-106. doi:10.1016/S0003-2670(97)00418-2

[24] S. Wold, M. Sjostrom and L. Eriksson, "PLS-Regression: A Basic Tool of Chemometrics,” Chemometrics and Intelligent Laboratory Systems, Vol. 58, No. 2, 2001, pp. 109-130. doi:10.1016/S0169-7439(01)00155-1

[25] E. Dinc, C. Yucesoy and F. Onur, "Simultaneous Spectrophotometric Determination of Mefenamic Acid and Paracetamol in a Pharmaceutical Preparation Using Ratio Spectra Derivative Spectrophotometry and Chemometric Methods," Journal of Pharmaceutical and Biomedical Analysis, Vol. 28, No. 6, 2002, pp. 1091-1100. doi:10.1016/S0731-7085(02)00031-6

[26] M. Bahram, "Mean Centering of Ratio Spectra as a New Method for Determination of Rate Constants of Consecutive Reactions," Analytica Chimica Acta, Vol. 603, No. 1, 2007, pp. 13-19. doi:10.1016/j.aca.2007.09.041

[27] A. Afkhami and M. Bahram, "Mean Centering of Ratio Spectra as a New Spectrophotometric Method for the Analysis of Binary and Ternary Mixtures,” Talanta, Vol. 66, 2005, pp. 712-720. doi:10.1016/j.talanta.2004.12.004 